\title{
Mass to Light Ratio, Initial Mass Function, and Chemical Evolution in Disk Galaxies
}

\author{
L. Portinari ${ }^{1,3}$, J. Sommer-Larsen ${ }^{1}$, and R. Tantalo ${ }^{2}$ \\ ${ }^{1}$ Theoretical Astrophysics Center, Juliane Maries Vej 30, DK 2100 Copenhagen $\varnothing$, Denmark \\ 2 Dipartimento di Astronomia, Università di Padova, Vicolo dell'Osservatorio 2, I 35122 Padova, Italy \\ ${ }^{3}$ E-mail: 1portina@tac.dk
}

Received 2003 September 26, accepted 2004 February 16

\begin{abstract}
Cosmological simulations of disk galaxy formation, when compared to the observed Tully-Fisher relation, suggest a low mass to light $(M / L)$ ratio for the stellar component in spirals. We show that a number of 'bottom-light' initial mass functions (IMFs) suggested independently in the literature, do imply $M / L$ ratios as low as required, at least for late type spirals (Sbc-Sc). However the typical $M / L$ ratio, and correspondingly the zero point of the Tully-Fisher relation, is expected to vary considerably with Hubble type.

Bottom-light IMFs tend to have a metal production in excess of what is typically estimated for spiral galaxies. Suitable tuning of the IMF slope and mass limits, post-supernova fallback of metals onto black holes or metal outflows must then be invoked, to reproduce the observed chemical properties of disk galaxies.
\end{abstract}

Keywords: galaxies: spiral — galaxies: evolution — stars: initial mass function

\section{Introduction}

Recent $N$-body + SPH (smoothed particle hydrodynamics) cosmological simulations of the formation of disk galaxies reproduce the observed Tully-Fisher (TF) relation (Dale et al. 1999), provided the mass to light $(M / L)$ ratio of the stellar component is rather low, $M / L_{\mathrm{I}}=0.7-1$ in the I band (Sommer-Larsen \& Dolgov 2001; Sommer-Larsen et al. 2003; Figure 1). The location of the simulated galaxies in the $\left(M_{*}, V_{c}\right)$ plane of Figure 1

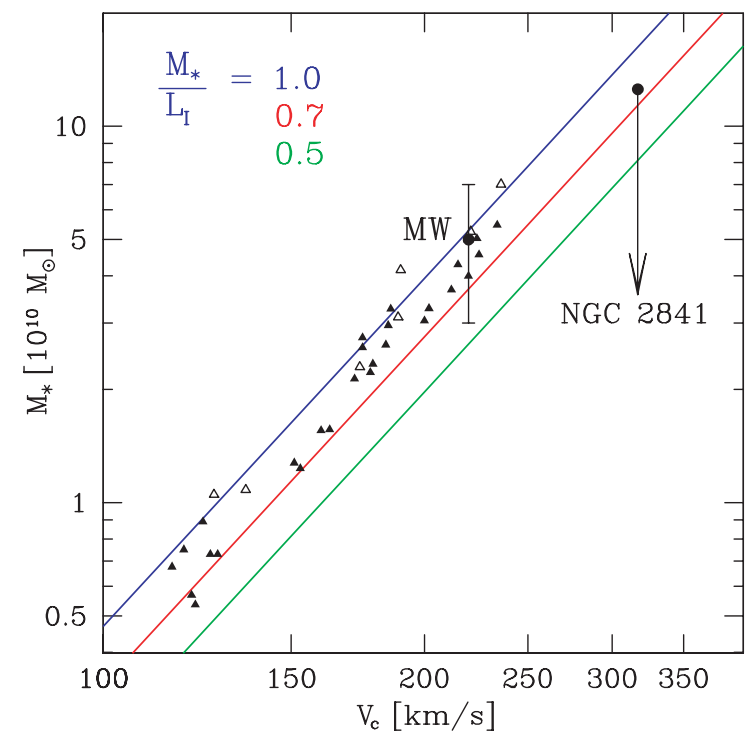

Figure 1 Straight lines: observed TF relation for Sbc-Sc disk galaxies (Dale et al. 1999; $h=0.7$ adopted here), for different assumptions about the stellar $M / L_{\mathrm{I}}$ ratio; triangles: simulated galaxies by Sommer-Larsen et al. (2003). Also shown is the location of the Milky Way and NGC 2841. is quite independent of the adopted initial mass function (IMF) or feedback efficiency: the baryonic mass that cools out to form a galactic disk and its resulting circular velocity correlate so that data points tend to move along the TF relation, hardly affecting the zero point (Navarro \& Steinmetz 2000a, 2000b). However, the IMF is crucial for the $M / L$ ratio, to translate the stellar masses $M_{*}$ to luminosities and compare the simulated TF relation to the empirical one. Although the zero point of the simulated TF may change with the concentration of the dark matter halos, and hence with the normalisation of the power spectrum $\sigma_{8}$ (Navarro \& Steinmetz 2000a, 2000b; Eke et al. 2001), many other arguments support a low stellar $M / L$ ratio in spiral galaxies.

The stellar mass of the Milky Way is $M_{*} \sim 5 \times 10^{10} \mathrm{M}_{\odot}$; to lie on the observed TF relation as do other spirals, its $M / L_{\mathrm{I}}$ value must be $\lesssim 1$ (Sommer-Larsen \& Dolgov 2001; Figure 1). A low $M / L_{\mathrm{I}}<0.8$ is also derived for the massive Sb galaxy NGC 2841, when compared to the observed TF relation (Portinari et al. 2004a, hereafter PST; Figure 1).

Based on bar instability arguments, Efstathiou et al. (1982) suggest an upper limit of $M / L_{\mathrm{B}} \leq 1.5 h$ for disks, i.e. $M / L_{\mathrm{B}} \lesssim 1$ for $h=0.7$ ( $h$ being the Hubble constant $\mathrm{H}_{0}$ in units of $\left.100 \mathrm{~km} \mathrm{sec}^{-1} \mathrm{Mpc}^{-1}\right)$.

The stellar $M / L$ ratio is related to the issue of whether disks are maximal or sub-maximal, i.e. whether or not they dominate the dynamics and rotation curves in the inner galactic regions. Even in the case of maximal stellar disks, $M / L$ ratios for the stellar component lower than those predicted by the Salpeter IMF are required (Bell \& de Jong 2001). And it is still much debated whether disks are maximal or sub-maximal; for his favoured sub-maximal disk model, Bottema (2002) finds $M / L_{\mathrm{I}} \sim 0.82$. 


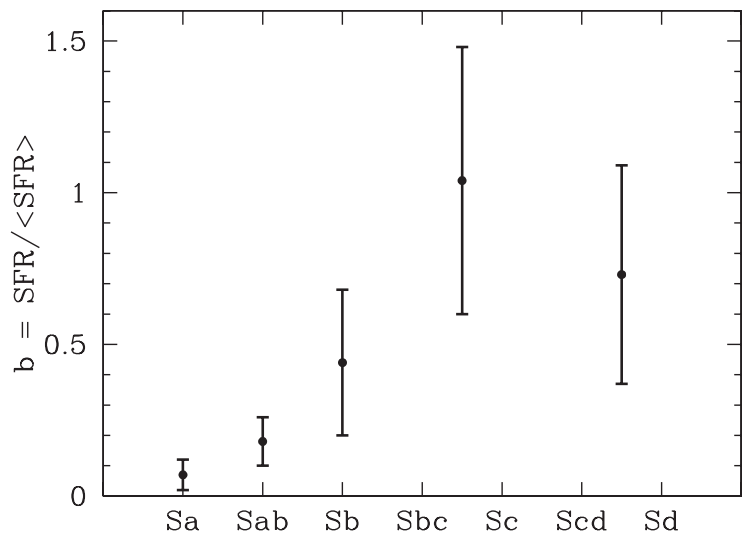

Figure 2 Mean $b$ parameter as a function of Hubble type. Data from Sommer-Larsen et al. (2003).

Finally, two recent dynamical studies of individual spiral galaxies yield $M / L \sim 1$ in the $\mathrm{B}, \mathrm{V}$, and $\mathrm{I}$ bands for the Sc galaxy NGC 4414 (Vallejo et al. 2002) and $M / L_{\mathrm{I}}=1.1$ for the disk of the Sab spiral $2237+0305$, Huchra's lens (Trott \& Webster 2002).

In this paper we discuss whether such low $M / L$ ratios are compatible with our understanding of stellar populations and chemical evolution in disk galaxies. We also address the effects of different star formation histories on the TF relation for different Hubble types.

\section{Star Formation History and Initial Mass Function}

The $M / L$ ratio of the stellar component of a galaxy (including both living stars and remnants) depends on the stellar IMF and on the star formation history (SFH) of the system.

The SFH of a disk galaxy is related to its Hubble type: Kennicutt et al. (1994) demonstrated that the sequence of spiral types is in fact a sequence of different SFHs in the disks, as traced by the birthrate parameter

$$
b=\frac{\mathrm{SFR}}{\langle\mathrm{SFR}\rangle}
$$

or the ratio between the present and the past average star formation rate (SFR); see Figure 2. Our reference TF relation by Dale et al. (1999) in Figure 1 is representative for Sbc-Sc spirals (Giovanelli et al. 1997). Kennicutt et al. (1994) find that Sbc-Sc disks correspond to $b=0.8-1$, hence we will concentrate our discussion on objects with SFHs corresponding to these values for $b$.

As for the IMF, a standard Salpeter slope extended over the mass range (0.1-100) $\mathrm{M}_{\odot}$ certainly yields much higher $M / L$ ratios than those mentioned in the Introduction (Figure 4, top left). There is, however, plenty of observational evidence that the IMF flattens below $\sim 1 \mathrm{M}_{\odot}$, possibly with a turnover at low masses, and hence is 'bottom-light' with respect to the Salpeter IMF.

We considered the following IMFs: the Salpeter (1955) IMF; the Kroupa (1998) IMF, derived from field stars in the solar neighbourhood; the Kennicutt (Kennicutt et al. 1994) IMF, derived from the global properties of spiral galaxies; the Larson (1998) IMF, with an exponential

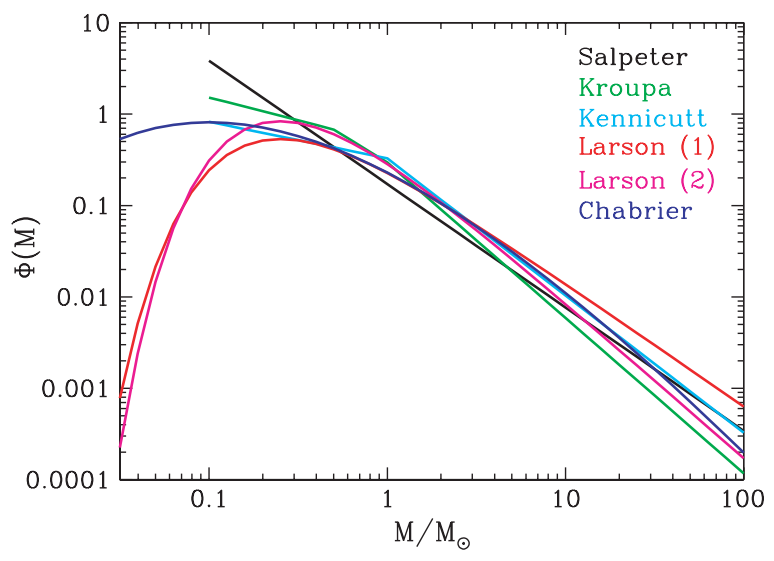

Figure 3 Comparison between the Salpeter IMF and the bottomlight IMFs considered in this paper, all normalised to the same total integrated mass of $1 \mathrm{M}_{\odot}$.

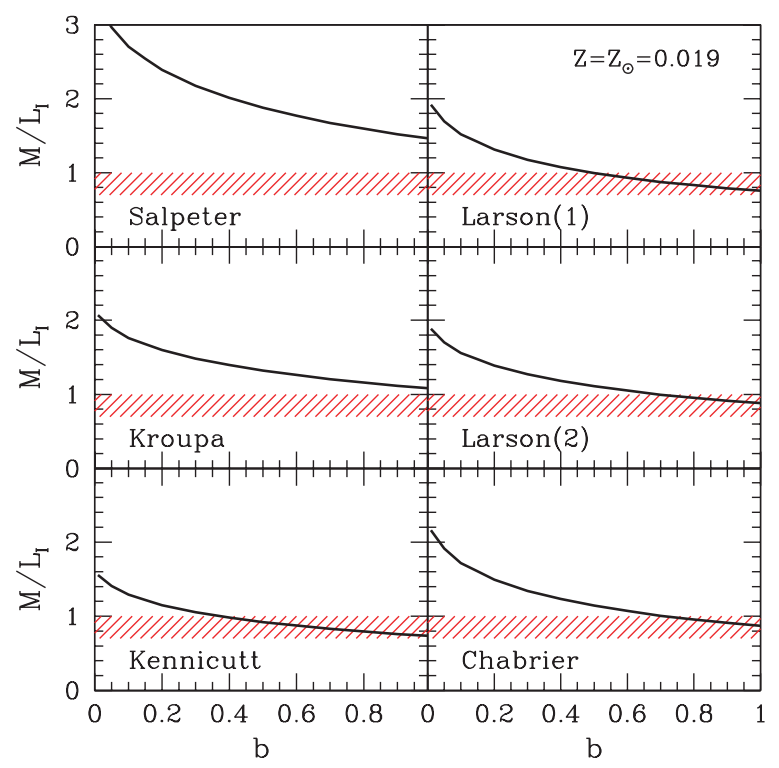

Figure $4 \mathrm{I}$ band $M / L$ ratio at varying $b$ parameter of the $\mathrm{SFH}$, for different IMFs. The red shaded area marks the range $M / L_{\mathrm{I}}=0.7-1$ favoured by observations for Sbc-Sc disks (corresponding to $b=0.8-1)$.

cutoff at low masses as favoured by recent determinations of the local IMF down to the subdwarf regime (Chabrier 2001, 2002); a modified Larson IMF, with the same peak mass but a steeper slope at high masses, in accordance with local determinations (Scalo 1986); and the Chabrier (2001, 2002) IMF, derived from low mass stars and brown dwarfs in the local field. With respect to Salpeter, all the other IMFs are bottom-light (Figure 3).

\section{Results from Simple Models}

We will focus on the $M / L$ ratio in the I band, which is an excellent optical tracer of stellar mass, because the I band luminosity is less sensitive to recent sporadic star formation and to corrections for dust extinction than bluer bands. Besides, the $M / L$ ratio of single stellar populations (SSPs) in the I band is less metallicity dependent than in other bands, and it is less sensitive to the specific treatment 
of the asymptotic giant branch phase than redder, nearinfrared bands (PST).

We computed SSPs based on the latest Padua isochrones (Girardi et al. 2002) for the different IMFs in Section 2. These are to be convolved with suitable SFHs. We generated a set of SFHs characterised by different values of the $b$ parameter, by adopting an exponentially decaying SFR $\propto \mathrm{e}^{-t / \tau}$ with different decaying rates. SSP metallicities around solar can be considered typical for spiral galaxies. The resulting $M / L$ ratio of the global stellar population (including remnants) as a function of the $b$ parameter is displayed in Figure 4 for the different IMFs. The range $0.8 \leq b \leq 1$ is representative of Sbc-Sc spirals. The red shaded area indicates the range in $M / L=0.7-1$ suggested in Section 1.

Figure 4 shows that bottom-light IMFs can in fact yield $M / L_{\mathrm{I}}<1$ for late-type spirals, though one probably needs slightly 'lighter' IMFs than the local Kroupa (1998) one.

\section{Offsets of the TF Relation with Hubble Type}

From Figure 4, the stellar $M / L$ ratio is expected to vary with Hubble type due to the differences in SFH parameterised by $b$. This effect implies systematic offsets with Hubble type of the luminosity zero point of the TF relation (Rubin et al. 1985; Giovanelli et al. 1997; Kannappan et al. 2002).

Figure 5 shows the $M / L$ ratio as a function of $b$, normalised to the value corresponding to $b=1$. The scale on the right axis indicates the corresponding shift in magnitude. With respect to Sbc-Sc spirals, we predict a systematic offset of $0.3-0.4$ mag for $\mathrm{Sb}(b \sim 0.35)$ and of $0.6-0.8 \mathrm{mag}$ for $\mathrm{Sa}-\mathrm{Sab}(b \sim 0.1)$, as a result of the different characteristic SFHs. These offsets are only slightly reduced when bulges are added to disks in the computation of the total $M / L$ ratios of galaxies (PST). The offsets we predict are larger than the empirical ones found by Giovanelli et al. (1997): $0.1 \mathrm{mag}$ for Sb spirals and $0.32 \mathrm{mag}$ for earlier types. However, the extent of the observed offsets in the TF relation is still a matter of debate: for instance, the larger offsets found by Kannappan et al. (2002) in the R band, 0.76 mag for Sa, are in good agreement with our predictions.

The characteristic SFH of a galaxy is traced by its colours, so that the offsets in $M / L$ ratio due to different SFHs can be corrected for, by applying suitable $M / L$ versus colour relations (Bell \& de Jong 2001; PST).

\section{Bottom-light IMFs and Chemical Evolution}

Besides simple models with exponentially declining SFHs, we also computed more realistic, multizone chemophotometric models of galactic disks, including infall, inside-out formation, and radially varying star formation efficiency. Chemical evolution is followed with the code by Portinari et al. (1998) and Portinari \& Chiosi (1999), and the models are calibrated to reproduce the typical metallicity and metallicity gradient of Sbc-Sc disks (PST). Six sets of models have been computed for the six IMFs in Section 2. The corresponding photometric properties are

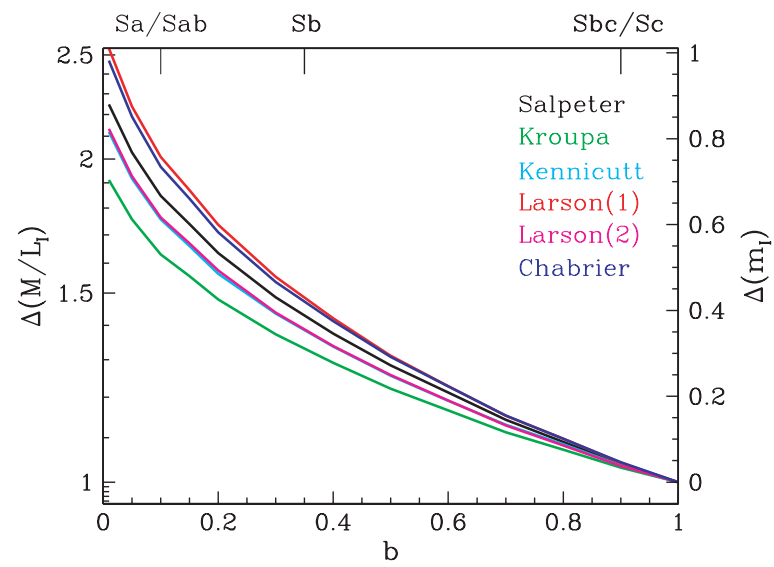

Figure 5 Relative $M / L$ ratio normalised to the value corresponding to $b=1$ models.

calculated by convolving the SFH and metal enrichment history of each annulus of the disk, with a grid of SSPs of metallicities between $5 \times 10^{-4} \mathrm{Z}_{\odot}$ and $5 \mathrm{Z}_{\odot}(\mathrm{PST})$.

For each IMF, the chemophotometric models confirm the $M / L$ ratios predicted, as a function of $b$, by the simple models in Figure 4; see the example for the Kennicutt IMF models in Figure 6, top panel. The results of Section 3 are thus confirmed: the bottom-light IMFs considered here imply $M / L_{\mathrm{I}}<1$ for Sbc-Sc spirals, as required in Section 1. This conclusion remains valid also when the contribution of the bulge to the global $M / L$ ratio is included (PST).

Chemophotometric models also allow an insight into the implications of bottom-light IMFs for chemical evolution. Some of the IMFs considered (Kennicutt, Larson, and Chabrier) are too efficient in metal production to reproduce the observed properties of spirals. In particular, the resulting gas fraction is much larger than the observed $M_{\text {gas }} / L_{\mathrm{B}} \sim 0.5 \mathrm{M}_{\odot} / \mathrm{L}_{\odot}$ (solid dots in Figure 6 , bottom panel). For a given IMF and corresponding metal production, the final gas metallicity predicted by chemical models increases with decreasing gas fraction (Tinsley 1980; Pagel 1997). Our models, calibrated to reproduce the observed metallicities, tend to predict gas fractions which are too high. Conversely, if they were calibrated to reproduce the observed gas fractions, they would result in metallicities which are too high.

This excessive metal production is readily understood since the enrichment efficiency of a stellar population, or its 'net yield', is inversely proportional to the mass fraction that remains forever locked in low-mass stars and remnants (Tinsley 1980; Pagel 1997); for bottom-light IMFs the locked-up fraction is small. This effect can be compensated by a steep slope at the high-mass end, which reduces the number of massive stars and the related metal production: the Kroupa or modified Larson IMFs, for instance, with a steep Scalo slope do not overproduce metals (see PST for details). A steep slope for the integrated field stars' IMF is expected, in fact, if stars form in star clusters of finite size even from an intrinsically shallower IMF (Kroupa \& Weidner 2003). 


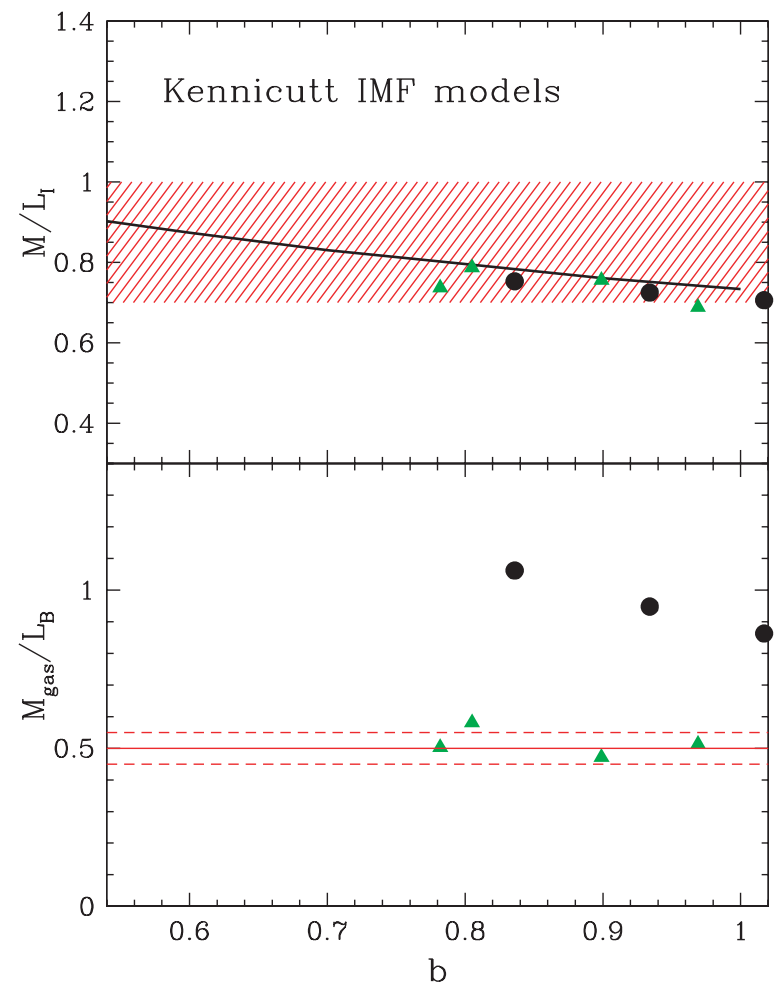

Figure 6 Top panel: $M / L$ ratio for chemophotometric models with the Kennicutt IMF (solid dots); they confirm the prediction from the simple exponential models (black line) and are within the observed range of $M / L_{\mathrm{I}}=0.7-1$ (red shaded area). Bottom panel: Predicted gas fractions compared to the observed range, marked by the red dashed lines. Green triangles: models with IMF mass limits tuned between $0.05-0.1 \mathrm{M}_{\odot}$ and $30-35 \mathrm{M}_{\odot}$, rather than the standard mass range $(0.1-100) \mathrm{M}_{\odot}$, to reproduce the observed gas fraction.

For those bottom-light IMFs that do imply an excess of metal production, one way to reconcile models with observations is to tune the upper mass limit of the IMF, again reducing the number of massive stars and related metal production - or equivalently assume that above a certain progenitor mass, the metals produced by a supernova fall back onto a black hole after the explosion. The observed gas fractions can then be matched without altering the stellar $M / L$ ratio significantly (green triangles in Figure 6).

Alternatively, we need to invoke substantial outflows of metals from disk galaxies into the intergalactic medium, to reconcile the high enrichment efficiency with the observed low gas fractions. This behaviour is reminiscent of that of elliptical galaxies, responsible for the enrichment of the hot gas in clusters.

With 'standard' IMFs suited to model the chemical evolution of the solar neighbourhood (e.g. the Kroupa IMF) it is impossible to account for the observed metal enrichment in clusters (Portinari et al. 2004b). Possibly, some of the bottom-light IMFs, advocated here to reproduce low disk $M / L$ ratios, suggest a scenario where the IMF and the enrichment efficiency may be the same in spiral and cluster galaxies, and in both cases much of the metals is dispersed into the intergalactic medium. However, substantial outflows would challenge our understanding of disk galaxy formation: in galactic disks, star formation proceeds at a smooth, non burst-like pace and the observed 'fountains' and 'chimneys' do not have enough energy to escape the galactic potential; winds are far less plausible than in spheroids. Moreover, strong ongoing stellar feedback and outflows could significantly hamper the dynamical formation of galactic disks from the cool-out of halo gas.

The alternative to major winds from disk galaxies is that the metal production in spirals is much lower than in galaxy clusters, because of a different IMF (Portinari et al. 2004b). The IMF may change out of Jeans mass variations with redshift (Moretti et al. 2003, and references therein); or be a universal function within star clusters, but lead statistically to more high-mass stars in massive ellipticals, where in regimes of intense star formation larger star clusters can be formed (Kroupa \& Weidner 2003).

\section{Acknowledgments}

L.P. is grateful to the organisers and to Swinburne University for generous hospitality during the GCD-V conference.

\section{References}

Bell, E. F., \& de Jong, R. S. 2001, ApJ, 550, 212

Bottema, R. 2002, A\&A, 388, 809

Chabrier, G. 2001, ApJ, 554, 1274

Chabrier, G. 2002, ApJ, 567, 304

Dale, D. A., Giovanelli, R., Haynes, M. P., Campusano, L. E., \& Hardy, E. 1999, AJ, 118, 1489

Efstathiou, G., Lake, G., \& Negroponte, J. 1982, MNRAS, 199, 1069

Eke, V. R., Navarro, J. F., \& Steinmetz, M. 2001, ApJ, 554, 114

Giovanelli, R., Haynes, M. P., Herter, T., \& Vogt, N. P. 1997, AJ, 113,53

Girardi, L., Bressan, A., Bertelli, G., Chiosi, C., Groenewegen, M. A. T., Marigo, P., Salasnich, B., \& Weiss, A. 2002, A\&A, 391, 195

Kannappan, S. J., Fabricant, D. G., \& Franx, M. 2002, AJ, 123, 2358

Kennicutt, R. C., Tamblyn, P., \& Congdon, C. W. 1994, ApJ, 435, 22

Kroupa, P. 1998, in Brown Dwarfs and Extrasolar Planets, eds. R. Rebolo, E. L. Martin, \& M. R. Zapatero Osorio, ASP Conf. Series 134 (San Francisco: ASP), 483

Kroupa, P., \& Weidner, C. 2003, ApJ, 598, 1076

Larson, R. B. 1998, MNRAS, 301, 569

Moretti, A., Portinari, L., \& Chiosi, C. 2003, A\&A, 408, 431

Navarro, J. F., \& Steinmetz, M. 2000a, ApJ, 528, 607

Navarro, J. F., \& Steinmetz, M. 2000b, ApJ, 538, 477

Pagel, B. E. J. 1997, Nucleosynthesis and Chemical Evolution of Galaxies (Cambridge: Cambridge University Press)

Portinari, L., \& Chiosi, C. 1999, A\&A, 350, 827

Portinari, L., Chiosi, C., \& Bressan, A. 1998, A\&A, 334, 505

Portinari, L., Sommer-Larsen, J., \& Tantalo, R. 2004a, MNRAS, 347, 691 (PST)

Portinari, L., Moretti, A., Chiosi, C., \& Sommer-Larsen, J. 2004b, ApJ, 604, 579

Rubin, V. C., Burstein, D., Ford, W. K., \& Thonnard, N. 1985, ApJ, 289, 81

Salpeter, E. E. 1955, ApJ, 121, 161

Scalo, J. M. 1986, FCPh, 11, 1

Sommer-Larsen, J., \& Dolgov, A. 2001, ApJ, 551, 608

Sommer-Larsen, J., Götz, M., \& Portinari, L. 2003, ApJ, 596, 47

Tinsley, B. M. 1980, FCPh, 5, 287

Trott, C. M., \& Webster, R. L. 2002, MNRAS, 334, 621

Vallejo, O., Braine, J., \& Baudry, A. 2002, A\&A, 387, 429 